\title{
Four-Dimensional Null Energy Condition as a Swampland Conjecture
}

\author{
Heliudson Bernardo, ${ }^{1, *}$ Suddhasattwa Brahma $\odot,{ }^{1, \dagger}$ Keshav Dasgupta $\odot,{ }^{1, \star}$ Mir Mehedi Faruk, ${ }^{1, \S}$ and $\operatorname{Radu} \operatorname{Tatar}^{2, \|}$ \\ ${ }^{1}$ Department of Physics, McGill University, Montréal, Quebec H3A 2T8, Canada \\ ${ }^{2}$ Department of Mathematical Sciences, University of Liverpool, Liverpool, L69 7ZL, United Kingdom
}

(Received 4 August 2021; revised 10 September 2021; accepted 29 September 2021; published 27 October 2021)

\begin{abstract}
We analyze four-dimensional Friedmann-Lemaître-Robertson-Walker cosmologies in type IIB, arising from a M-theory dual, and find that the null energy condition (NEC) has to be obeyed by them (except for the negatively curved case) in order for the M-theory action to have a Wilsonian effective description. However, this does not imply that the M-theory metric has to obey the 11D NEC. Thus, we propose a new swampland conjecture - the 4D NEC is a consistency condition for any theory to have a completion within M theory-with an explicit derivation of it for cosmological backgrounds from a top-down perspective. We briefly discuss the cosmological consequences of such a condition derived from $\mathrm{M}$ theory.
\end{abstract}

DOI: 10.1103/PhysRevLett.127.181301

Introduction.-Energy conditions are considered important for constraining physically viable solutions of Einstein's equations. In particular, the null energy condition (NEC) plays a crucial role in cosmology and is a key ingredient for proving the Hawking-Penrose singularity theorems [1]. The NEC implies that the matter stressenergy tensor should satisfy

$$
T_{\mu \nu} l^{\mu} l^{\nu} \geq 0
$$

for a lightlike vector $l^{\mu}$. On assuming general relativity (GR), one finds the Ricci convergence condition $R_{\mu \nu} l^{\mu} l^{\nu} \geq 0$. Although the NEC seems to be a reasonable restriction, there is no compelling derivation of it from fundamental theory [2] (see, however, [3-8] for some preliminary attempts in this direction). On the other hand, there are many effective field theories (EFTs) which can violate the NEC and a priori, there does not seem to be a good reason to banish them [9-11]. Since these theories lead to compelling cosmological model building with interesting physical implication (e.g., see [12-16]), finding a foundational origin of the NEC, as a physically necessary condition, would have profound consequences. For instance, bouncing cosmologies-solutions which posit that an expanding universe is created from a previously collapsing one-present an alternative to the standard cosmological paradigm [17] and typically require NEC violation [18-21]. The goal of this Letter is to show a surprising link between the 4D NEC and a general

Published by the American Physical Society under the terms of the Creative Commons Attribution 4.0 International license. Further distribution of this work must maintain attribution to the author(s) and the published article's title, journal citation, and DOI. Funded by SCOAP ${ }^{3}$. consistency condition emanating from M theory, thereby ruling out such bouncing solutions in string theory.

Although supergravity theories, which are low-energy limits of string theory, have stress-energy tensors which do obey some of the energy conditions, there is no general expectation that energy conditions have to be satisfied in string theory due to some inherent fundamental reason. In fact, there exist all kinds of higher curvature terms, quantum corrections, and other stringy objects (such as orientifolds and branes) which indicate that energy conditions can easily be violated in string theory. Generally speaking, the role of energy conditions in string theory is also quite well known. The strong energy condition has been used to derive no-go theorems for having 4D de Sitter space descending from low-energy supergravity actions (in the absence of quantum corrections) in higher dimensions $[22,23]$. On the other hand, some of the stringy effects mentioned above [24], as well as time-dependent internal dimensions, allow one to bypass this and find accelerating cosmologies (see, for instance, [25,26] or [27] for a review). What is clear, however, is the key role energy conditions play in understanding the types of cosmological solutions which are allowed in string theory ([28,29] presents a recent overview). Keeping this in mind, our main objective is to arrive at a remarkable derivation of the four-dimensional NEC starting from $\mathrm{M}$ theory. We will derive a condition which comes from requiring that $\mathrm{M}$ theory has a well-defined EFT description and show that it has precisely the same form as the NEC in 4D. Crucially, this will not imply that the NEC has to be satisfied in full (higher-dimensional) $\mathrm{M}$ theory but is only a consequence for the external spacetime.

Recently, there has been a considerable effort in identifying universal features of quantum gravity which would help us in demarcating consistent EFTs in 4D that have a UV completion, namely, the swampland program [30-32]. 
Our work takes a significant stride in this direction by identifying a top-down condition from $\mathrm{M}$ theory which requires that any consistent $4 \mathrm{D}$ EFT containing gravity must satisfy the NEC in order to find an embedding in $\mathrm{M}$ theory. In this way, we find a compelling reason to elevate the status of the 4D NEC to a swampland conjecture-a necessary condition that any 4D EFT has to satisfy in order to have a UV completion within string theory. This shall have a lot of striking consequences for many cosmological models in 4D. In particular, an important implication of this is that since it is well known that violating the NEC is a necessary condition for the existence of bounces in flat Friedmann-Lemaitre-Robertson-Walker (FLRW) cosmologies, we show that such bounces cannot arise in theories descending from $\mathrm{M}$ theory with a well-defined Wilsonian effective action, supporting previous similar claims from other considerations [33-36].

Let us sketch our main result which can be understood as follows. One starts with an 11D M-theory metric which allows for a 4D FLRW spacetime and a time-dependent (warped) internal spacetime (in the dual type IIB side) and includes all types of (time-dependent) fluxes and local and nonlocal quantum corrections (including higher curvature terms) that are needed to support such a spacetime [37-39]. We shall then derive a necessary condition for all these quantum terms to have a hierarchy, so as to have a welldefined Wilsonian effective action, which will impose a constraint on the allowed form of $a(t)$ for the external dimensions [40]. Naturally, allowing such flux sources, stringy extended objects and quantum corrections imply that the higher-dimensional metric does not obey simple two-derivative Einstein's equations. Nevertheless, one can still put all the corrections and sources to the right-hand-side of Einstein's equations and work with an effective 10D stress-energy tensor which supports such a metric. What we find, quite remarkably, is that the condition required for the quantum terms to maintain their hierarchy is exactly the same as the NEC for the 4D external metric in the dual IIB side. Thus, as long as one has a well-defined EFT description for the fluxes and quantum terms included in the M-theory action, the 4D FLRW metric will automatically obey the $N E C$. What is more is that the 4D NEC does not imply that the higher-dimensional metric obeys the 10D NEC, the latter condition not expected to arise from string theory.

The NEC from $M$ theory.-Let us come straight to the most novel part of our argument. On the M-theory side, let us take a metric ansatz of the form [44]

$$
\begin{aligned}
d s^{2}= & \frac{e^{2 A(y, \eta)}}{f^{1 / 3}(r, \theta)}\left(-d \eta^{2}+g_{i j} d x^{i} d x^{j}\right) \\
& +\frac{e^{2 B(y, \eta)}}{f^{1 / 3}(r, \theta)} \tilde{g}_{m n} d y^{m} d y^{n} \\
& +e^{2 C(y, \eta)} f^{2 / 3}(r, \theta)\left(\frac{d \phi^{2}}{g_{b}^{2}}+d x_{11}^{2}\right),
\end{aligned}
$$

with $g_{b}$ being the type IIB string coupling (which is kept at the constant coupling point in $\mathrm{F}$ theory), $(m, n=4, \ldots, 9)$ and $(i, j=1,2)$ and where

$$
f(r, \theta)=\frac{1}{r^{2} \sin ^{2} \theta}, \quad g_{11}=\frac{1}{1-k r^{2}}, \quad g_{22}=r^{2} .
$$

Although unfamiliar, this form of the metric in M theory simply assumes a general FLRW metric for 4 external dimensions in the dual IIB side, for an 11D space which has the topology of $\mathcal{M}_{11}=\mathbb{R}^{2,1} \times \mathcal{M}_{6} \times \mathbb{T}^{2} / \mathcal{G}$, where $\tilde{g}_{m n}(y, \eta)$ is the unwarped metric of the $6 \mathrm{D}$ base and $\mathcal{G}$ is the isometry group. Although, as we show below and as alluded to above, there is a type IIB metric corresponding to (2), we begin with this uplifted metric as it shall help us in identifying the time dependence of the IIA string coupling which will be useful for organizing the time dependence of all the quantum corrections and the flux components [45]. The warping factor $[\mathrm{H}(y)]$ is contained in the expressions:

$$
\begin{aligned}
e^{2 A} & =g_{b}^{-2 / 3} a(\eta)^{\frac{8}{3}} \mathrm{H}(y)^{-\frac{8}{3}} \\
e^{2 B} & =g_{b}^{-2 / 3} a(\eta)^{\frac{2}{3}} \mathrm{H}(y)^{\frac{4}{3}} \\
e^{2 C} & =g_{b}^{4 / 3} a(\eta)^{-\frac{4}{3}} \mathrm{H}(y)^{\frac{4}{3}},
\end{aligned}
$$

where $a(\eta)$ is the usual scale factor for the 4D cosmological metric. Dimensionally reducing the $x_{11}$ direction, we get

$$
\begin{aligned}
d s^{2}= & e^{2 A(y, \eta)+C(y, \eta)}\left(-d \eta^{2}+g_{i j} d x^{i} d x^{j}\right) \\
& +e^{2 B(y, \eta)+C(y, \eta)} \tilde{g}_{m n}(y, \eta) d y^{m} d y^{n}+e^{3 C(y, \eta)} f(r, \theta) \frac{d \phi^{2}}{g_{b}^{2}},
\end{aligned}
$$

with time-dependent type IIA coupling $g_{s} \equiv e^{3 C / 2} f^{1 / 2}$. In fact, we will use $g_{s}$ to represent the temporal behavior in the M-theory side. Finally, $T$-dualizing the $\phi$ direction, we get a type IIB metric of the form [46]

$$
\begin{aligned}
d s^{2}= & \frac{a^{2}(\eta)}{\mathrm{H}^{2}(y)}\left(-d \eta^{2}+g_{i j} d x^{i} d x^{j}\right. \\
& \left.+r^{2} \sin ^{2} \theta d \phi^{2}\right)+\mathrm{H}^{2}(y) \tilde{g}_{m n}(y, \eta) d y^{m} d y^{n} .
\end{aligned}
$$

As is clear from the discussion above, $y$ collectively denotes the internal spatial directions for us. Interestingly, as shown in $[38,39]$, we need to allow for time-dependent fluxes for supporting such a configuration which results in a timedependent $\tilde{g}_{m n}$. However, we shall still require that the 4D Newton's constant $G_{N}$ remains fixed. One can further split up the internal 6D manifold $\mathcal{M}_{6}=\mathcal{M}_{4} \times \mathcal{M}_{2}$, and separate out the time dependence of it, to get 


$$
d s^{2}=\frac{1}{\mathrm{H}^{2}(y)} d s_{\mathrm{FLRW}}^{2}+\mathrm{H}^{2}(y)\left[F_{1}(\eta) d s_{\mathcal{M}_{2}}^{2}+F_{2}(\eta) d s_{\mathcal{M}_{4}}^{2}\right],
$$

where the unwarped metrics corresponding to the internal metrics $d s_{\mathcal{M}_{2}}^{2}$ and $d s_{\mathcal{M}_{4}}^{2}$ are now time independent. In this more familiar form, the external spacetime can be clearly seen to be a FLRW cosmology and the condition to have $G_{N}$ constant implies that we additionally require $F_{1} F_{2}^{2}=1$, and both $F_{1,2}(\eta) \rightarrow 1$ as $g_{s} \rightarrow 0$. Note that the $(2,4)$ splitting of the internal manifold, while convenient, is not essential. One could have other splittings like $(1,5),(3,3)$, or even $\left(a_{1}, a_{2}, \ldots\right)$ with $a_{1}+a_{2}+\ldots=6$ as long as the internal six-volume remains time independent and, in the limit $g_{s} \rightarrow 0$, remains nonsingular.

Assuming the scale factor to be of the form $a(\eta) \sim \Lambda^{n / 2} \eta^{n}$, the type IIA coupling takes the form

$$
g_{s}=\frac{g_{b} \mathrm{H}(y)}{\left(\Lambda \eta^{2}\right)^{n / 2} r \sin \theta} .
$$

Note that the late time regime is weakly coupled: $g_{s} \rightarrow 0$. The important new condition required for having a welldefined hierarchy to the higher curvature and quantum terms is that time derivatives of $g_{s}$ should always be given in terms of non-negative powers of $g_{s}$, i.e.,

$$
\frac{d g_{s}}{d \eta} \propto g_{s}^{(1+1 / n) \geq 0} \Rightarrow \frac{1}{n} \geq-1 .
$$

This is the crucial condition for us, the detailed derivation of which from the M-theory side can be seen in Sec-3 of [47] (and a brief outline in the Supplemental Material [48]). The main argument behind this condition is that for the dominant $g_{s}$ scaling of the various terms to be positive, so as to maintain a hierarchy between the different quantum and higher curvature terms, implies that the time derivative of $g_{s}$ must be a positive power of $g_{s}$. A heuristic way to understand this condition (11) is that, for our analysis, we not only require that the type IIA coupling remains small so that we are in the weak-coupling limit, but also that it remains small for the regime of validity of the solution. Therefore, (11) ensures that both $g_{s}$ and its time-derivative remains small in this regime.

Having derived the key condition (11), it is easy to show that this is the NEC for a 4D flat $(k=0)$ FLRW cosmology in disguise. We shall consider the open and closed case $(k= \pm 1)$ later on. For a perfect fluid in 4D, the NEC condition is given by $\rho+p \geq 0$, where $\rho$ and $p$ are the energy and pressure densities, respectively. Assuming Einstein's equations (or, in other words, considering an effective stress-energy tensor) for a flat, FLRW spacetime, it is easy to show that the NEC implies that $\dot{H} \leq 0$ where we denote the Hubble parameter as $H(t)=\dot{a} / a$, written in terms of cosmic time. On assuming a power-law ansatz, $a(t) \propto t^{\gamma}$, the NEC implies $\gamma \geq 0$. Converting to conformal time, as was done for the metric (8) above, i.e., $a(\eta) \propto \eta^{\gamma /(1-\gamma)}=: \eta^{n}$, the NEC for the 4D $k=0$ FLRW metric takes the form $(1 / n) \geq-1$, which is exactly the same as (11).

No NEC for IIB metric.-Let us go back to (9) and calculate the Riemann and the Ricci components for this metric, to demonstrate that

$$
R_{00}^{(10 d)}+a^{-2} R_{11}^{(10 d)}=-2 \dot{H}-3 \frac{\dot{F}_{2}^{2}}{F_{2}^{2}},
$$

where we have assumed $F_{1} F_{2}^{2}=1$, as required. [Other splittings of the internal six-manifold introduce different positive powers of $F_{i}$ in (12).] If the IIB metric were to obey the NEC (in 10D), then we would get the condition

$$
-2 \dot{H}-3 \frac{\dot{F}_{2}^{2}}{F_{2}^{2}} \geq 0 .
$$

However, recall that (11) simply implies that $-\dot{H} \geq 0$, and therefore we cannot comment whether (9) obeys the NEC or not. Note that this conclusion is only dependent on our requirement that the $4 \mathrm{D} G_{N}$ remains time-independent and does not depend on the details of the splitting of the internal manifold. This is a very intriguing finding and let us comment on its physical implication. Requiring that there exists a hierarchy in the various flux, curvature, and quantum terms included in the M-theory action-as is necessary to support a metric of the form (2) -implies that the external 4D metric has to obey the NEC. But this does not imply that the higher-dimensional metric also has to obey the NEC. Physically, this is indeed what one could have expected. Since we are allowing all sorts of higher curvature and (local and nonlocal) quantum corrections, along with time-dependent $G$-flux sources, our equations are very far away for the low-energy supergravity ones. Thus, there is no reason to expect that our effective stressenergy tensor for M theory obeys any energy condition, including the NEC. Moreover, the higher-dimensional NEC would impose a geometric restriction which would never be reproduced from the lower-dimensional spacetime since there are higher-dimensional null vectors which have vanishing components in some of the external directions. Also, note that for a given dimension, having a Wilsonian effective action does not imply anything like the NEC at all. In fact, there are well-known quantum field theories involving higher derivative terms which violate the NEC but have a consistent EFT description [10,11]. What we do find is that requiring that the 11D M theory has all of its terms under control, in the sense of having a well-defined hierarchy of terms in the effective action, automatically leads to imposing the NEC on the external 4D flat FLRW cosmology. This is why our result is exactly the same in the spirit of the "swampland" conjectures - we find that a large space of 4D Lagrangians (all those which violate the 4D NEC) cannot find a UV-completion into M theory. 
Advantages and assumptions. - At this point, let us emphasize the main assumptions in our derivation above. First, we assume that the external FLRW spacetime is parametrized by $a(\eta) \propto \eta^{n}$. This is true for perfect fluids in $4 \mathrm{D}$ with a constant equation of state of the form $p=w \rho$. Second, we require that the M-theory action must have hierarchy between quantum corrections of different orders and, therefore, has a well-defined Wilsonian EFT description. Although this is a rather conservative assumption, it might happen that there exists solutions for which one needs to take into account quantum corrections of all orders and no truncations to any finite order is allowed. Third, although we allow for time-dependent fluxes and internal dimensions, we make sure that $G_{N}$ remains constant. And finally, for our explicit calculations, we have kept the type IIB dilaton to be time independent although this is not a significant limitation and it will not be too difficult to relax this in the future.

Having said this, note that our analysis provides a powerful advantage over other approaches and it is rather general in the following sense. Our M-theory solution is not limited to leading order in $\alpha^{\prime}$ or $g_{s}$ corrections. Indeed, we allow for all types of perturbative, nonperturbative, and topological quantum corrections along with all possible higher-curvature terms. We find that time-dependent fluxes are necessary to support a configuration like (2), which has a 4D external FLRW metric, so those have to be included as well. This is, in fact, what should make us skeptical about whether the higher-dimensional metric would obey anything like the NEC. More importantly, this implies that we do not constrain the effective stress-energy tensor for our M-theory solution to obey any energy conditions. Simply ensuring that there exists a hierarchy between the different terms allows us to derive (11), which turns out to be the NEC for the 4D flat FLRW metric.

Cosmological implications.-An immediate consequence of (11) is that cosmological bounces are ruled out for flat FLRW spacetimes if they have to descend from $\mathrm{M}$ theory. This is not a statement for bounces in the context of classical gravity. Indeed, we are deriving a condition (11) from full $\mathrm{M}$ theory which happens to match with the NEC for $k=0$ FLRW cosmology. We emphasize that we are constraining an effective stress-energy tensor for the external 4D metric, the full embedding of which within M theory (2) contains all types of flux sources and quantum corrections. Thus, we are not just considering the avoidance of singularity by some classical bouncing solution but rather commenting on the status of bouncing cosmologies having a UV completion within string theory. There have been previous similar statements regarding banishing cosmological bounces from principles of holography [33] or properties of initial or final boundary conditions [34]. However, our argument comes from a much more general principle and is therefore, applicable to a much wider class of cosmological models. The fact that we have to obey the 4D NEC does not, of course, mean that singularity resolution is not possible in cosmological models of M theory. One can think of a situation where the bouncing solution requires corrections to all orders and so has no effective description [52], or even where spacetime is emergent from more fundamental UV degrees of freedom [53].

The second important conclusion of having to obey the NEC is that the Hubble parameter can neither grow today nor in the early universe (superinflation) [54]. More specifically, models of dark energy which violate the NEC would be ruled out immediately insofar that they can have no quantum gravity completion. A large class of dark energy models which require an equation of state $w<-1$ is immediately ruled out. This is of enormous phenomenological importance since the recent Hubble tension is seemingly alleviated by dark energy models which have a phantom component [55,56] and our condition (11) would severely disfavor such Lagrangians [57].

Further consequences of having the NEC as a swampland condition is that it would rule out traversable Lorentzian wormholes in 4D [58,59] and creating a universe in a laboratory [60]. Moreover, all NEC violating FLRW cosmologies, such as what one gets from a large subclass of modified gravity (for instance, from Horndeski or, more generally, DHOST) theories, are ruled out due to this consistency condition. Therefore, we are able to severely constrain the space of allowed cosmological models which come from a plethora of $4 \mathrm{D}$ gravitational theories, if they are to have a UV-complete description.

Let us end our discussion of cosmological implications with an important disclaimer. It is important to emphasize that our condition (11) only exhibits the NEC for 4D and not the strong energy condition (SEC). Had we found the latter, it would have ruled accelerating solutions in cosmology for M theory. However, as discussed in [37,38], we do find such solutions, including 4D de Sitter space since, as stressed above, we do not require that the M-theory stress-energy tensor satisfies the 11D NEC. It has been independently shown that satisfying the SEC, even if the NEC is violated, allows for time-depending compactifications to 4D de Sitter space [28].

Generalization to $k= \pm 1$. - Note that our condition (11), which comes from $\mathrm{M}$ theory, is true for any curvature (2). It just so happens that this coincides with the NEC for the flat FLRW metric in 4D. In order to see what our condition implies for the closed $(k=1)$ and the open $(k=-1)$ case, let us write down the NEC for a general FLRW metric. Assuming $a(t) \sim t^{\gamma}$, as before, we find the NEC implies

$$
-\frac{d^{2}}{d t^{2}}\left(\ln t^{\gamma}\right)+k t^{-2 \gamma} \geq 0 \Rightarrow \gamma+k t^{2(1-\gamma)} \geq 0 .
$$

This confirms our previous assertion that the NEC for $k=0$ is $\gamma \geq 0$, which written in terms of scale factor 
expressed in conformal time translates to (11). The above equation (14) immediately tells us that given (11), the NEC is also going to be automatically satisfied for closed FLRW spacetime. This is because, for the $k=1$ case, the second term in (14) is necessarily positive. Finally, for the negatively curved $k=-1$ case, (11) does not imply the NEC condition in this case. To summarize, we find that our new swampland conjecture would be that any EFT in a 4D closed or flat FLRW cosmology must satisfy the NEC in order to have a UV completion.

A final point to note is that this also indicates that our analysis rules out cosmological bounces only for flat FLRW spacetimes. This is so because violating the NEC is a necessary condition for having a bounce only for flat and open FLRW metrics (and we do not get the NEC for the open case). In fact, although condition (11) implies the NEC for the closed case, one can have cosmic bounces for this geometry without violating the NEC. Therefore, our conclusions regarding bounces are only limited to the $k=0$ FLRW spacetime. Having said this, let us note that a generic contracting solution is unstable against anisotropies and can even become strongly inhomogeneous due to the Belinski-Khalatnikov-Lifshitz conjecture. To avoid this stability problem of bounces, a typical attractor solution is often invoked known as ekpyrosis [61,62], which assumes a superstiff equation of state $w \gg 1$. However, what this physically implies is that near the bounce, at the end of the contracting phase, the term proportional to the curvature $k$ is subleading and therefore, for ekpyrotic scenarios, our results would generically apply. In other words, although we have a condition which explicitly rules out cosmological bounces for flat FLRW spacetimes, in effect we find a very strong argument against all types of bounces since a contracting solution that is stable against anisotropies is agnostic about the curvature of spacetime anyway.

Conclusion.-In this Letter, we showed that there appears a remarkable connection between the requirement of having a well-defined Wilsonian EFT for M theory (with a time-dependent compactification) and the NEC in four dimensions. This led us to conclude that we can rule out bounces in M theory, at least for flat FLRW cosmologies. Since we explicitly derive the NEC only for $k=0$ and $k=1$ FLRW spacetimes starting from M theory, in the spirit of the swampland, we conjecture the following: Any 4D consistent theory of gravity must obey the NEC in order to have an embedding in $\mathrm{M}$ theory. We emphasize that our conjecture, for the specific 4D FLRW backgrounds mentioned above, is actually derived from $\mathrm{M}$ theory without any bottom-up considerations.

S. B. is supported in part by the NSERC (funding reference No. CITA 490888-16) through a CITA National Fellowship and by a McGill Space Institute fellowship. H. B., K. D., and M.M.F. are supported in part by the NSERC grants. *heliudson@hep.physics.mcgill.ca

†suddhasattwa.brahma@gmail.com

"keshav@hep.physics.mcgill.ca

§mir.faruk@mail.mcgill.ca

"Radu.Tatar@Liverpool.ac.uk

[1] S. W. Hawking and R. Penrose, The Singularities of gravitational collapse and cosmology, Proc. R. Soc. A 314, 529 (1970).

[2] E. Curiel, A primer on energy conditions, Einstein Stud. 13, 43 (2017).

[3] M. Parikh and J. P. van der Schaar, Derivation of the null energy condition, Phys. Rev. D 91, 084002 (2015).

[4] C. de Rham and S. Melville, Unitary null energy condition violation in $\mathrm{P}(\mathrm{X})$ cosmologies, Phys. Rev. D 95, 123523 (2017).

[5] M. Parikh and A. Svesko, Thermodynamic origin of the null energy condition, Phys. Rev. D 95, 104002 (2017).

[6] I. Sawicki and A. Vikman, Hidden negative energies in strongly accelerated Universes, Phys. Rev. D 87, 067301 (2013).

[7] D. A. Easson, I. Sawicki, and A. Vikman, When matter matters, J. Cosmol. Astropart. Phys. 07 (2013) 014.

[8] D. A. Dobre, A. V. Frolov, J. T. G.Ghersi, S. Ramazanov, and A. Vikman, Unbraiding the bounce: Superluminality around the corner, J. Cosmol. Astropart. Phys. 03 (2018) 020.

[9] V. A. Rubakov, The null energy condition and its violation, Phys. Usp. 57, 128 (2014).

[10] N. Arkani-Hamed, H. C. Cheng, M. A. Luty, and S. Mukohyama, Ghost condensation and a consistent infrared modification of gravity, J. High Energy Phys. 05 (2004) 074.

[11] A. Nicolis, R. Rattazzi, and E. Trincherini, Energy's and amplitudes' positivity, J. High Energy Phys. 05 (2010) 095; 11 (2011) 128(E).

[12] M. Visser and C. Barcelo, Energy conditions and their cosmological implications, arXiv:gr-qc/0001099.

[13] L. Alberte, P. Creminelli, A. Khmelnitsky, D. Pirtskhalava, and E. Trincherini, Relaxing the cosmological constant: A proof of concept, J. High Energy Phys. 12 (2016) 022.

[14] P. Creminelli, M. A. Luty, A. Nicolis, and L. Senatore, Starting the Universe: Stable violation of the null energy condition and non-standard cosmologies, J. High Energy Phys. 12 (2006) 080.

[15] T. Qiu, Y. F. Cai, and X. M. Zhang, Null energy condition and dark energy models, Mod. Phys. Lett. A 23, 2787 (2008).

[16] I. Y. Aref'eva and I. V. Volovich, On the null energy condition and cosmology, Theor. Math. Phys. 155, 503 (2008).

[17] R. Brandenberger and P. Peter, Bouncing cosmologies: Progress and problems, Found. Phys. 47, 797 (2017).

[18] C. Molina-Paris and M. Visser, Minimal conditions for the creation of a Friedman-Robertson-Walker universe from a 'bounce', Phys. Lett. B 455, 90 (1999).

[19] Y. F. Cai, T. Qiu, Y. S. Piao, M. Li, and X. Zhang, Bouncing universe with quintom matter, J. High Energy Phys. 10 (2007) 071.

[20] C. Lin, R. H. Brandenberger, and L. P.Levasseur, A matter bounce by means of ghost condensation, J. Cosmol. Astropart. Phys. 04 (2011) 019.

[21] T. Qiu, J. Evslin, Y. F. Cai, M. Li, and X. Zhang, Bouncing galileon cosmologies, J. Cosmol. Astropart. Phys. 10 (2011) 036. 
[22] G. W. Gibbons, Aspects of supergravity theories, in Supersymmetry, Su- pergravity and Related Topics, edited by F. del Aguilla, A. Azcarrage, and L. E. Ibanez (World Scientific, Singapore, 1985).

[23] J. M. Maldacena and C. Nunez, Supergravity description of field theories on curved manifolds and a no go theorem, Int. J. Mod. Phys. A 16, 822 (2001).

[24] S. B. Giddings, S. Kachru, and J. Polchinski, Hierarchies from fluxes in string compactifications, Phys. Rev. D 66, 106006 (2002).

[25] S. Kachru, R. Kallosh, A. D. Linde, and S. P. Trivedi, De Sitter vacua in string theory, Phys. Rev. D 68, 046005 (2003).

[26] S. Kachru, R. Kallosh, A. D. Linde, J. M. Maldacena, L. P. McAllister, and S. P. Trivedi, 'Towards inflation in string theory, J. Cosmol. Astropart. Phys. 10 (2003) 013.

[27] M. Cicoli, S. De Alwis, A. Maharana, F. Muia, and F. Quevedo, De Sitter vs Quintessence in string theory, Fortschr. Phys. 67, 1800079 (2019).

[28] J. G. Russo and P. K. Townsend, Time-dependent compactification to de Sitter space: A no-go theorem, J. High Energy Phys. 06 (2019) 097.

[29] J. G. Russo and P. K. Townsend, Late-time cosmic acceleration from compactification, Classical Quantum Gravity 36, 095008 (2019).

[30] T. D. Brennan, F. Carta, and C. Vafa, The string landscape, the swampland, and the missing corner, Proc. Sci., TASI2017 (2017) 015 [arXiv:1711.00864].

[31] E. Palti, The swampland: Introduction and review, Fortschr. Phys. 67, 1900037 (2019).

[32] M. Graña and A. Herráez, The swampland conjectures: A bridge from quantum gravity to particle physics, Universe 7, 273 (2021).

[33] N. Engelhardt and G. T. Horowitz, Holographic consequences of a no transmission principle, Phys. Rev. D 93, 026005 (2016).

[34] G. T. Horowitz and J. M. Maldacena, The black hole final state, J. High Energy Phys. 02 (2004) 008.

[35] B. Craps, T. Hertog, and N. Turok, On the quantum resolution of cosmological singularities using AdS/CFT, Phys. Rev. D 86, 043513 (2012).

[36] T. Hertog and G. T. Horowitz, Holographic description of AdS cosmologies, J. High Energy Phys. 04 (2005) 005.

[37] S. Brahma, K. Dasgupta, and R. Tatar, Four-dimensional de Sitter space is a Glauber-Sudarshan state in string theory, J. High Energy Phys. 07 (2021) 114.

[38] K. Dasgupta, M. Emelin, M. M. Faruk, and R. Tatar, de Sitter vacua in the string landscape, Nucl. Phys. B969, 115463 (2021).

[39] K. Dasgupta, M. Emelin, M. M. Faruk, and R. Tatar, How a four-dimensional de Sitter solution remains outside the swampland, J. High Energy Phys. 07 (2021) 109.

[40] In fact, time-dependence and Wilsonian effective action require the M-theory configuration to be realized as a Glauber-Sudarshan state and the corresponding fluctuations as an Agarwal-Tara state [37,41] (see also [42,43]). We will, however, not deal with these subtleties here.

[41] S. Brahma, K. Dasgupta, and R. Tatar, de Sitter space as a Glauber-Sudarshan state, J. High Energy Phys. 02 (2021) 104.
[42] G. Dvali, C. Gomez, and S. Zell, Quantum break-time of de Sitter, J. Cosmol. Astropart. Phys. 06 (2017) 028.

[43] G. Dvali, C. Gomez, and S. Zell, Quantum breaking bound on de Sitter and swampland, Fortschr. Phys. 67, 1800094 (2019).

[44] Throughout, $\eta$ and $t$ denotes conformal and cosmic time, respectively.

[45] There is another deeper reason for using M-theory uplift: the IIB configuration is at a constant coupling point, and $g_{b}=1$ with vanishing axio-dilaton. This means it is at strong coupling (where $S$ duality doesn't help). M-theory uplift is the only way to allow for a controlled laboratory for the IIB computations.

[46] Note that $e^{2 A+C}=g_{b}^{2} e^{-3 C}=\left[a^{2}(\eta) / \mathrm{H}^{2}(y)\right]$ and $e^{2 B+C}=$ $\mathrm{H}^{2}(y)$.

[47] H. Bernardo, S. Brahma, K. Dasgupta, M. M. Faruk, and R. Tatar, de Sitter space as a Glauber-Sudarshan state: II, arXiv:2108.08365 [Fortschr. Phys. (to be published)].

[48] See Supplemental Material at http://link.aps.org/ supplemental/10.1103/PhysRevLett.127.181301 for a detailed derivation of the our condition (11) from M-theory, which includes Refs. [49-51].

[49] K. Dasgupta, M. Emelin, E. McDonough, and R. Tatar, Quantum corrections and the de Sitter swampland conjecture, J. High Energy Phys. 01 (2019) 145.

[50] K. Becker, M. Becker, and A. Strominger, Five-branes, membranes and nonperturbative string theory, Nucl. Phys. B456, 130 (1995).

[51] H. Bernardo, S. Brahma, K. Dasgupta, and R. Tatar, Crisis on infinite earths: Short-lived de Sitter vacua in the string theory landscape, J. High Energy Phys. 04 (2021) 037.

[52] J. Quintin, H. Bernardo, and G. Franzmann, Cosmology at the top of the $\alpha^{\prime}$ tower, J. High Energy Phys. 07 (2021) 149.

[53] N. Seiberg, Emergent spacetime, arXiv:hep-th/0601234.

[54] M. Baldi, F. Finelli, and S. Matarrese, Inflation with violation of the null energy condition, Phys. Rev. D 72, 083504 (2005).

[55] E. Di Valentino, A. Melchiorri, and J. Silk, Reconciling Planck with the local value of $H_{0}$ in extended parameter space, Phys. Lett. B 761, 242 (2016).

[56] S. Vagnozzi, New physics in light of the $H_{0}$ tension: An alternative view, Phys. Rev. D 102, 023518 (2020).

[57] E. Ó. Colgáin and M. M. Sheikh-Jabbari, A critique of holographic dark energy, Classical Quantum Gravity 38, 177001 (2021).

[58] M. Visser, Lorentzian Wormholes: From Einstein to Hawking (AIP, Woodbury, USA, 1995).

[59] M. S. Morris and K. S. Thorne, Wormholes in space-time and their use for interstellar travel: A tool for teaching general relativity, Am. J. Phys. 56, 395 (1988).

[60] E. Farhi and A. H. Guth, An obstacle to creating a Universe in the laboratory, Phys. Lett. B 183, 149 (1987).

[61] J. Khoury, B. A. Ovrut, P. J. Steinhardt, and N. Turok, The Ekpyrotic universe: Colliding branes and the origin of the hot big bang, Phys. Rev. D 64, 123522 (2001).

[62] J. Khoury, B. A. Ovrut, N. Seiberg, P. J. Steinhardt, and N. Turok, From big crunch to big bang, Phys. Rev. D 65 , 086007 (2002). 